\title{
S26. Sleep disorders: epidemiology, social impact and treatment attitudes (supported by an educational grant from Synthélabo F)
}

\begin{abstract}
Burden of Psychological Problems in General Health Care: WHO Collaborative Project

T.B. Üstün on behalf of all the Participating Investigators * World Health Organization, Division of Mental Health, Geneva, Swizerland

OBJECTIVES: This paper reports the burden of psychological illness in general health care a WHO collaborative study of the "Psychological Problems in General Health Care".

METHODS: This project was carried out in 14 countries in different parts of world. Research methods are described in detail in Arch Gen Psychiatry Vol 50, pp 819-824, Oct 1993.

STUDY RESULTS: Psychological problems are frequent in alt the general health care settings examined in this study i.e depressive disorders, anxiety disorders, alcohol use disorders, and somatoform disorders. All these disorders were associated with marked disability. The presence of psychological disorder was associated with substantial levels of disability among patients studies in general health care settings as measured by malfuntion in daily activities and occupational roles; activity limited days. These findings were consistent across diverse countries and cultures.

CONCLUSION: The psychological problems of general health care patients constitute a major public health problem worldwide because: they are frcquent and have significant burden on the society. Specific action has to be taken to improve identification and managemnt of these problems.

* Investigators from participating centres are: O. Öztürk M. Rezaki (Ankara); C. Stefanis, V. Mavreas (Athens); S.M. Channabasavanna, T.G. Sriram (Bangalore); H. Helmchen, M. Iinden, (Berlin); W. van den Brink, B. Tiemens, (Groningen); M. Olatawura, O. Gureje (badan); O. Benkert, W. Maier (Mainz); D. Goldberg, R. Gater (Manchester); Y. Nakane, M. Michitsuji (Nagasaki); Y. Lecrubier, P. Boyer (Paris); J A. Costa e Silva, L. Villano (Seatte); Yan He Qin, Xaio Shi Fu (Shanghai); M. Tansella, C. Bellantuono (Verona)

** Advisory Group : J.A. Costa e Siva, D. Goldberg, M. Von Korf, Y. Lecrubier, H. Ormel, H.U. Wittchen, T.B. Üstün (Project Coordinator), N. Sartorius (chair).
\end{abstract}

\author{
FORM AND FREQUENCY OF SLEEP PROBLEMS IN \\ GENERAL HEALTH CARE \\ Y. Lecrubier, E. Weiller \\ INSERM U302 - Hôpital La Salpêtrière - Pav. Clérambault - 47, bd de \\ l'Hôpital - 75013 Paris - France.
}

In 1990-1992 the World Health Organisation organised a large epidemiological study in primary care aimed to describe in 14 countries the psychological problems encountered in general health care. The study used a two-stage case finding procedure. Out of about 26000 consecutive patients briefly described, 5438 passed a second stage interview comprising the primary care version of the CIDI, an assessment of disability, the recognition of the patients as a psychiatric case by GPs and the treatment proposed.

In this study psychological disorders were found to be common in primary care : $20.3 \%$ of patients presented with a current ICD-10 diagnosis. Additionally $8.5 \%$ had subthreshold diagnosis paralleled by a substantial disability. $30.8 \%$ of primary care attenders were only symptomatic ( $\leq 2$ psychological symptoms). Insomnia was one of the commonest symptoms: $24.5 \%$ among 5416 patients. Two third of those with an ICD-10 diagnosis $(n=1238)$ complained of any insomnia, the figures were $44.8 \%$ for subthreshold psychological disorders, $25.5 \%$ for symptomatic patients and $2.7 \%$ for the remaining patients. The prevalence of insomnia according to the number of diagnosis increased from $60 \%$ for those with a single diagnosis to $85 \%$ of those with 4 or more diagnosis. Early insomnia and night awakenings were more frequent than early awakening. For those with an ICD-10 diagnosis the figures were respectively $48.7 \%$, $43 \%$ and $38 \%$. Almost $50 \%$ of patients complained of difficulties falling asleep irrespective to their diagnostic status (depressive episode, dysthymia, GAD, agoraphobia, panic disorder, somatoform disorder or hypochondriasis). Patients with agoraphobia complained of early morning awakenings $(45 \%)$ more than depressed $(33.7 \%)$ and GAD patients $(27.5 \%)$. Treatment strategies will be described. 
ClASSIC AND NOVEL HYPNOTIC DRUGS: SIMILARITIES AND DIFFERENCES

MG Terzano, L Parrino

Sleep Disorders Center, Dept. of Neurology, University of Parma, Italy

It is widely accepted that the conventional polysomnographic parameters (macrostructure of sleep) supply only rough information for clinical purposes. In particular, they often appear inadequate to support a diagnosis of insomnia or the efficacy of a hypnotic compound. In the past years, attention has been focused on the microstructure of sleep, and especially on the periodic distribution of arousal-related phasic events known as Cyclic Alternating Pattern (CAP). This microstructural thythm is not only a physiological component of normal NREM sleep, but it also appears highly sensitive in the detection of disturbing factors and drug manipulation. Regardless of the specific context, $C A P$ always translates a condition of arousal instability during sleep. Accordingly, the higher the amount of CAP, the poorer the subjective quality of sleep. In young adults, the phsyiological amount of CAP Rate (percentage ratio of CAP time to NREM sleep time) ranges around $25 \%$, while CAP Rate rises to $55 \%$ when sleep is perturbed by continuous white noise (situational insomnia). Therapeutical doses of zolpidem preserve the regular course of sleep both at the macro- and at the microstructural level, when sleep is recorded under basal conditions. In contrast, during acoustic perturbation, zolpidem reduces the pathological amounts of arousal instability by lowering the values of CAP Rate to $38 \%$. The protective activity of zolpidem against noise reverberates especially upon sleep stages 3 and 4 , where the disruptive effects of acoustic perturbation are more prominent. The results of this model of situational insomnia have been confirmed in a study carried out on a group of middleaged insomniacs in which CAP Rate appeared as the only polysomnographic parameter that permitted to discriminate patients from age-matched controls and the zolpidem nights from the placebo nights. The model of situational insomnia based on continuous noise and CAP analysis has been used for a multi-drug comparison in a group of middle-aged healthy subjects without complaints about sleep. All the assessed sedative-hypnotic agents (zolpidem, zopiclone, triazolam, lorazepam) reduce to different degrees the eccessive amounts of arousal instability induced by noise. In particular, zolpidem 10 mg restores physiological values of CAP Rate.

PRACTICAL EXPERIENCE WITH ZOLPIDEM IN PSYCHIATRIC PATIENTS: Mogens Undén

Sct. Hans Hospital, Department M, 4000 Roskilde, Denmark.

Most clinical trials on hypnotics exclude patients suffering from addiction, alcoholism, servere psychiatric disorders including dementia and serious systemic diseases (1). A study (2) has shown good efficacy on sleep-induction and maintenance of zolpidem as compared to flunitrazepam in 42 psychiatric patients. Clinical "naturalistic" experience has revealed, that zolpidem is an effective an helpful hypnotic in insomniac patients suffering from dementia (SDAT, vascular dementia and Chorea Huntington)

Likewise schizophrenic out-patients on stable neuroleptic monotherapy suffering from transient insomnia have shown good effect of zolpidem treatment. The same has been the case in patients suffering from major depression. Patients with depression or anxiety and cormorbid eventually symptomatic alcohol abuse and - dependence, have been clinically evaluated in out-patient alcoholism treatment clinics and the findings are of importance. During the period of abstinence - and postabstinence syndrome (up to 10 days), zolpidem seems to be less effective than unselective hypnotics e.g. benzodiazepines. This seems to be an important notion, because later on zolpidem is a fully effective hypnoctic in these patients.

From clinical experience it is concluded, that zolpidem is not abie to suppress physical abstinence symptoms, which may be a reflection of its Omega-I selectivity and the finding, that zolpidem in contrast to classical benzodiazepines and other non selective hypnotics is not able to induce phvsical dependence and thereby kindling in animal models (3).

1. Licardello L. and Licini P. Current Ther. Res., 1992,52.

2. Frattola L. et al: Double Blind Comparison of Zolpidem

$20 \mathrm{mg}$ versus Flunitrazepam $2 \mathrm{mg}$ in insomniac In-patients. Drugs Expt1. C1 in. Res., 1990, XVI ,7,371-376.

3. VonVoigtlander F.F., Lewis R.A: A Rapid Screening Method for assissment of Benzodiazepine Receptor-Related Physi cal Dependence in Mice. J. Pharmacol. Methods, 1991,26, $1-5$.

\section{SLEEP DISORDERS: FUTURE STRATEGIES FOR INSOMIA MANAGEMENT \\ Jj López-Ibor, Jr. \\ Psychiatric Department. Complutense University. Martín} Lagos s/n. 28035, Madrid, Spain

Insomnia is a symptom of many psychiatric and nonpsychiatric disorders. It also appears during non-pathological stressful situations. In recent times sleep research has been able to describe several forms of primary sleep disorders which in ICD-10 are differentiated from so called nonorganic sleep disorders. This differentiation is not always clear. Sleep disorders have to be considered in the context of the changes ot the sleep-wake cycle. For the clinician it is important for the management of sleep disorders the following steps: 1) The diagnosis of the nature of the sleep disorder itself and its repercussion on the sleep-wakefulness cycle. 2) The possible etiology of the sleep disorder. 3) The impact of a specific medication, specially psychotropic drugs on sleep and sleep-wakefulness cycle. A careful history taking and examination will lead to a diagnosis in most of the cases which will not need more in depth examinations, such as polisomnographic studies. Some hygienic measures and the treatment of underlying conditions such as depression may lead to a normalization of sleep. Sometimes specific hypnotics could be prescribed but here the question of tolerance and dependance play a role. Newer drugs for the treatment of insomia have in common a half-life shorter than the duration of normal sleep and this may lead to mantain a better sleep architecture during most of the night sleep, something which may have important repercussions for how patients with insomnia should be treated. 